\title{
Composite Reweighting the Glasgow Method for Finite Density QCD
}

\author{
P. R. Crompton ${ }^{\text {a }}$ \\ ${ }^{\text {a}}$ Dept. of Physics and Astronomy, University of Glasgow, G12 8QQ, Scotland, UK.
}

The reweighting scheme developed in Glasgow to circumvent the lattice action becoming complex at finite density suffers from a pathological onset transition thought to be due to the reweighting. We present a new reweighting scheme based on this approach in which we combine ensembles to alleviate the sampling bias we identify in the polynomial coefficients of the fugacity expansion.

\section{Introduction}

Lattice simulations of dense matter are important for the understanding of future heavy ion collider experiments as well as the equation of state of neutron stars [1] 2]. The main obstacle to the numerical evaluation of QCD in this regime is that the lattice action becomes complex with the inclusion of the chemical potential $\mu$, prohibiting naive probabilistic Monte Carlo methods. Recent attempts to evaluate QCD at finite baryon density have involved evaluating canonical ensembles with a given number of background quark sources [3], and the evaluation of QCD-like models in which the lattice action is real at finite $\mu$ [5] [6]. An earlier reweighting approach was developed at Glasgow, in which the Grand Canonical Partition function is evaluated semi-analytically.

\section{The Glasgow Method}

The $\mu$ dependence of the lattice action is made analytic in the Glasgow reweighting method through the formulation of a characteristic polynomial in the fugacity variable $z=\exp (\mu / T)$ [7. The fermion matrix $M$ (defined with KogutSusskind fermions [8]) is re-expressed in terms of the matrices which contain only links between lattice sites in the spatial directions $G$, and forward and backward in the time direction $V$ and $V^{\dagger}$. which allows the definition of the propagator matrix $P[9]$.

$$
\begin{aligned}
& 2 i M=2 i m+G+V e^{\mu}+V^{\dagger} e^{-\mu} \\
& P=\left(\begin{array}{cc}
-(G+2 i m) & 1 \\
-1 & 0
\end{array}\right) V
\end{aligned}
$$

The propagator matrix is then used to reexpress $\operatorname{det} M$ as a characteristic polynomial in the variable $e^{-\mu}$,

$$
\begin{aligned}
\operatorname{det} M & =\operatorname{det}\left(G+2 i m+V^{\dagger} e^{-\mu}+V e^{\mu}\right) \\
& =e^{n_{c} n_{s}^{3} n_{t} \mu} \operatorname{det}\left(P-e^{-\mu}\right) \\
& =e^{n_{c} n_{s}^{3} n_{t} \mu} \sum_{n=0}^{2 n_{c} n_{s}^{3} n_{t}} c_{n} e^{-n \mu}
\end{aligned}
$$

where $n_{s}^{3} n_{t}$ is the lattice volume, $n_{c}$ the number of colours, and the expansion coefficients $c_{n}$, so defined, are functionals of the lattice gauge fields. Since $V$ is an overall factor of $P$ the expansion can be further simplified through the symmetry $Z_{n_{t}}$ associated with performing a unitary transformation on $P$ by multiplying the timelinks by $e^{2 \pi i j / n_{t}}$, where $j$ is an integer. This then allows relation of the expansion coefficients of the characteristic polynomial (where $n_{t}=1 / T$ ) to the canonical partition functions $Z_{n}$ and the Grand Canonical Partition function $Z(\mu)$. As Lee and Yang showed with an Ising ferromagnetic system, in the thermodynamic limit a phase transition occurs wherever a zero of the fugacity polynomial approaches the real axis in the complex- $z$ plane [10]. The zeros $\alpha_{n}$ are determined numerically by rootfinding the reweighted polynomial expansion coefficients.

$$
\begin{aligned}
Z(\mu) & =\int D U \operatorname{det} M(\mu) e^{-S_{g}} \\
& =\sum_{n} Z_{n} e^{n \mu / T}
\end{aligned}
$$




$$
\begin{aligned}
\frac{Z(\mu)}{Z\left(\mu_{o}\right)} & =\frac{\int D U \frac{\operatorname{det} M(\mu)}{\operatorname{det} M\left(\mu_{o}\right)} \operatorname{det} M\left(\mu_{o}\right) e^{-S_{g}}}{\int D U \operatorname{det} M\left(\mu_{o}\right) e^{-S_{g}}}(8) \\
& =\left\langle\frac{\operatorname{det} M(\mu)}{\operatorname{det} M\left(\mu_{o}\right)}\right\rangle_{\mu_{o}} \\
& \propto e^{-n_{c} n_{s}^{3} n_{t} \mu} \prod_{n=1}^{n_{c} n_{s}^{3}}\left(e^{n_{t} \mu}-\alpha_{n}\right)
\end{aligned}
$$

Reweighting introduces normalisation by the ensemble generated at $\mu_{o}$ into Eqn.(9), which leaves the analytic determination of the critical points unaffected. The advantage of the scheme is that, even though the fermionic action is in general complex in $\mathrm{SU}(3)$, an ensemble can be generated at $\mu_{o}=0$. However, it has been shown that the unphysical onset transition at $\mu=\frac{1}{2} m_{\pi}$ of quenched measurements [11], persists despite the inclusion of dynamical quarks. From which it is concluded that the Monte Carlo sampling is ineffective, because the $\mu_{o}=0$ ensemble has little overlap with the physically relevant region 12] 13. Parallels can also be drawn between this pathology and the sign problem of the related reweighting of the Hubbard model [14].

\section{SU(2) at Intermediate Coupling}

\subsection{Composite Reweighting}

For $\mathrm{SU}(2)$ we can vary the value of $\mu_{o}$ we use to generate ensembles (as the group is pseudoreal for quarks in the fundamental representation [15]). This then allowed us to investigate the reliability of the ensemble-averaging of the polynomial expansion coefficients of Eqn.([), which is defined through the fugacity expansion.

$$
\begin{aligned}
\frac{Z_{n}}{Z\left(\mu_{o}\right)} & =\frac{\int D U \frac{c_{n}}{\operatorname{det} M\left(\mu_{o}\right)} \operatorname{det} M\left(\mu_{o}\right) e^{-S_{g}}}{\int D U \operatorname{det} M\left(\mu_{o}\right) e^{-S_{g}}}(11) \\
& =\left\langle\frac{c_{n}}{\operatorname{det} M\left(\mu_{o}\right)}\right\rangle_{\mu_{o}}
\end{aligned}
$$

These coefficients are evaluated recursively from the propagator matrix $P$, and the denominator $\operatorname{det} M\left(\mu_{o}\right)$ by evaluating Eqn.(5) at $\mu=\mu_{o}$. Where the ratio on the lefthand side of Eqn.(12)

\begin{tabular}{|c|c|c|c||}
\hline$\mu_{o}$ & $\operatorname{Re} \eta_{1}$ & $\operatorname{Im} \eta_{1}$ & $\mu\left(\max \chi_{n}\right)$ \\
\hline 0.3 & $0.411(0.001)$ & $0.116(0.001)$ & $0.41(0.01)$ \\
\hline 0.5 & $0.830(0.002)$ & $0.167(0.096)$ & $0.83(0.01)$ \\
\hline 0.7 & $0.523(0.003)$ & $0.134(0.001)$ & $0.52(0.01)$ \\
\hline 0.8 & $0.822(0.028)$ & $0.154(0.082)$ & $0.82(0.01)$ \\
\hline 0.9 & $0.546(0.067)$ & $0.153(0.051)$ & $0.55(0.01)$ \\
\hline 1.0 & $0.434(0.039)$ & $0.091(0.039)$ & $0.43(0.01)$ \\
\hline 1.1 & $0.461(0.011)$ & $0.064(0.030)$ & $0.46(0.01)$ \\
\hline$\#$. Ens. & - & - & - \\
\hline 1 & $0.546(0.067)$ & $0.153(0.051)$ & $0.55(0.01)$ \\
\hline 3 & $0.467(0.008)$ & $0.012(0.007)$ & $0.47(0.01)$ \\
\hline 5 & $0.453(0.008)$ & $0.011(0.007)$ & $0.45(0.01)$ \\
\hline 7 & $0.477(0.001)$ & $0.006(0.005)$ & $0.48(0.01)$ \\
\hline
\end{tabular}

Table 1

Dependence of $\eta_{1}$ for SU(2) at intermediate coupling on the $\mu_{o}$ (upper), and number of ensembles included in the composite reweighting (lower).

differs from one, we found from our measurements that the reliability of the ensemble-averaging is strongly affected. In turn this affected our determination of the Lee Yang zeros through rootfinding, and our measurement of related thermodynamic observables evaluated from the expansion coefficients. We monitored the effect by measuring the ratio $W^{21}(n)$, of the expansion coefficients from ensembles generated at two different values of $\mu_{o}$.

$$
\begin{aligned}
W^{21}(n) & =\left\langle\frac{c_{n}}{\operatorname{det} M\left(\mu_{2}\right)}\right\rangle_{\mu_{2}}\left\langle\frac{c_{n}}{\operatorname{det} M\left(\mu_{1}\right)}\right\rangle_{\mu_{1}}^{-1}(13) \\
& =\frac{Z\left(\mu_{1}\right)}{Z\left(\mu_{2}\right)}
\end{aligned}
$$

Although this ratio should be independent of $n$, our measurements indicate that ensembleaveraging selectively affects the determination of the expansion. Only a small range of $n$ can be reliably determined from an ensemble generated at a given value of $\mu_{o}$. However, since this small range centers on different $n$ as $\mu_{o}$ is varied, coefficients from a covering of several ensembles (generated at different values of $\mu_{o}$ ) can be combined to alleviate the bias inherent in the reweighting. 
The reliably ensemble-averaged range of coefficients from the ensemble generated at $\mu_{2}$ (multiplied by $W^{21}$ ) is used to replace the corresponding range of the expansion of the ensemble generated at $\mu_{1}$. As the number of covering ensembles is increased these new composite reweighted coefficients, which replace the ill-determined coefficients, thus converge to accurate values.

\subsection{Results}

Measurements of the chiral $\langle\bar{\psi} \psi\rangle$ and diquark condensates $\langle\psi \psi\rangle$ at strong [16] and intermediate coupling 17] 18] 19], indicate at least two distinct finite density regimes. The first, a low density vacuum regime in which $\langle\bar{\psi} \psi\rangle \neq 0\langle\psi \psi\rangle=0$, is believed to be separated from a thermodynamic regime, where $\langle\bar{\psi} \psi\rangle=0\langle\psi \psi\rangle \neq 0$, by a transition at $\mu=\frac{1}{2} m_{\pi}$. The $U(2)$ symmetry at $m, \mu=0$ allows a rotation between the two condensates and so the superfluid diquark can be used as an indicator of the spontaneous breaking of the staggered fermion chiral symmetry remnant $U(1)_{A}$ (in addition to $\left.U(1)_{V}\right)$.

Our measurements of the zeros of the Grand Canonical Partition function identify this transition as we tabulate in Table 1. By evaluating the Lee Yang zeros $\alpha_{n}$ in the complex $\mu$ plane we can readily identify the value of $\mu$ at the transition. In the thermodynamic limit $\operatorname{Im} \eta_{1} \rightarrow 0$ and so $\operatorname{Re} \eta_{1}$ corresponds to $\mu_{c}$.

$\eta_{n}=T \ln \alpha_{n}$

Before our composite reweighting procedure is applied, $\eta_{1}$ is inconsistent and strongly dependent on the value of $\mu_{o}$ used in the reweighting. However the jacknife error estimates with our new scheme indicate that the zeros can be consistently determined where we alleviate the inaccuracies caused by reweighting. As we increase the number of ensembles generated at succesive values of $\mu_{o}$ used to cover the expansion, $\operatorname{Re} \eta_{1}$ becomes consistent. Also $\operatorname{Im} \eta_{1} \rightarrow 0$, from which we are able to more confidently associate $\operatorname{Re} \eta_{1}$ with $\mu_{c}$. Our measurements at $\beta=1.5, m=0.05$ on a $6^{3} 4$ lattice indicate a transition at $\mu=0.48$, which is in approximate agreement with existing measurements 15]21] 22 23] 24. The prominent peaking of our further measurements of the quark

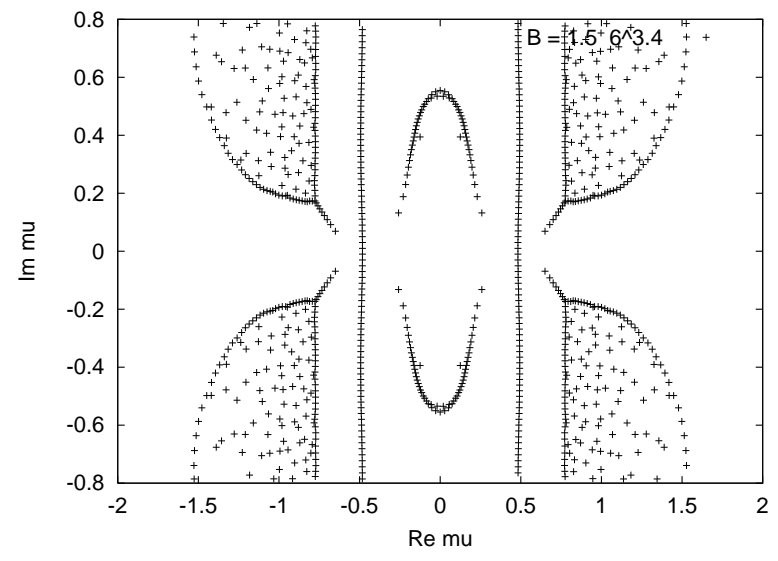

Figure 1. Lee Yang zeros evaluated in the complex $\mu$ plane for $\mathrm{SU}(2)$ at intermediate coupling from seven composite reweighted ensembles.

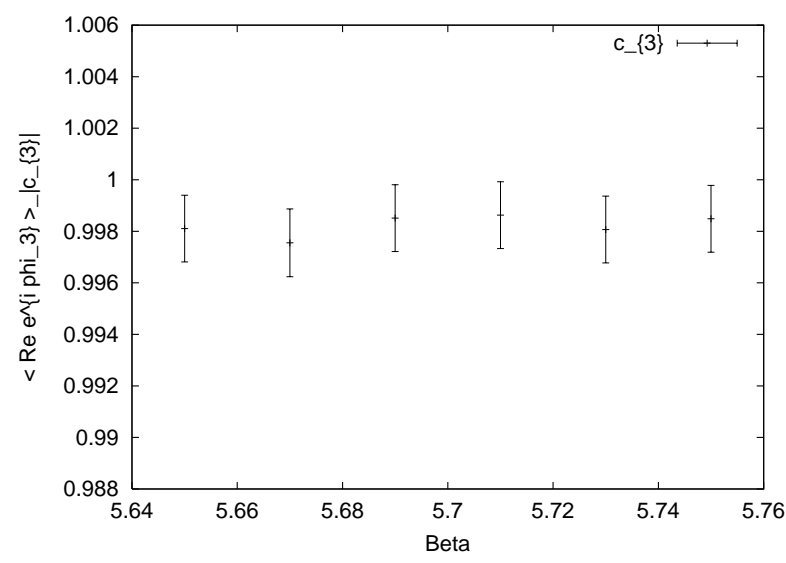

Figure 2. $\left\langle\operatorname{Re}\left(e^{i \phi_{n}}\right)\right\rangle_{\left|c_{n}\right|}$ the ensemble-averaged real part of the phase of the polynomial expansion coefficient $c_{n}$ (for $n=3$ ), which we use as a Monte Carlo measure for static SU(3) at intermediate coupling. 
number density susceptibility from the composite weighted expansion coefficients, we believe, indicates that this transition is first order.

\section{Static SU(3) at Intermediate Coupling}

Having developed our new approach in $\mathrm{SU}(2)$, we now evaluate the ensemble-averaged polynomial expansion coefficients of static SU(3) 25, from canonical ensembles generated with different numbers of background quark sources. In this way we are able to again systematically vary the localised region in which the ensemble-averaging is reliable, and combine coefficients from different ensembles.

$$
\begin{aligned}
\langle\mathcal{O}\rangle_{Z_{n}} & =\langle\mathcal{O}\rangle_{c_{n}} \\
& =\frac{\int D U \mathcal{O} c_{n} e^{-S_{g}}}{\int D U c_{n} e^{-S_{g}}} \\
& =\frac{\left\langle\mathcal{O} e^{i \phi_{n}}\right\rangle_{\left|c_{n}\right|}}{\left\langle e^{i \phi_{n}}\right\rangle_{\left|c_{n}\right|}}
\end{aligned}
$$

Our implementation of static SU(3) involves setting $G=0$ in Eqn.(2) which differs slightly from existing schemes [25] 26] [27] in that we additionally incorporate the relativistic effect of antiquarks. The motivation for choosing this model over full $\mathrm{SU}(3)$ is purely the computational expediency that refreshing $c_{n}$ in one dimension alone affords the Monte Carlo sampling. Although the expansion coefficients are in general complex, our measurements of the ensemble-averaged real part of the phase of our measure indicate that the sign problem of the measure is negligible, Fig.(2). As $n$ moves away from the index of the expansion coefficient used as the Monte Carlo measure, however, the ensemble averaging of the expansion coefficients becomes less effective, as we monitor in Fig.(3). For the two ensembles generated with the measures $c_{75}$ and $c_{51}, W^{75}{ }^{51}(n)$ is only reliably determined for $50<n<65$. Elsewhere error estimates indicate that the measurement is unreliable, and so only the expansion coefficients in this small range are effectively sampled. Our measurements at $\beta=5.71, m=0.1$ with a $6^{3} 4$ lattice volume of the zeros Fig.(4), and corresponding quark number density susceptibility, indicate a first order finite density transition at $\mu=0.098(1)$.

\section{Conclusions}

Although we have addressed the pathologies of the Glasgow method with our new composite reweighting approach, the main stumbling block to the evaluation of finite density $\mathrm{SU}(3)$ with dynamical fermions would appear to remain the numerical effort required to evaluate the reweighting method. We seem to have traded the overlap problem for the efficiency our evaluation of canonical ensembles, which we believe now requires parallel computing. However, rootfinders can often find the zeros of a polynomial from the first few coefficients of an expansion, which we can achieve in our method by implementing a shift variable to truncate the polynomial [28]. Further progress in evaluating $\mathrm{SU}(3)$ with dynamical quarks at finite density can therefore be made by evaluating ensembles weighted with the first few expansion coefficients alone in this manner.

Thanks to M. Alford, and I. M. Barbour for useful discussions.

\section{REFERENCES}

1. H. Satz Nucl. Phys. Proc. Suppl. 94, 204 (2000).

2. H. Heiselberg, V. Pandharipande Annu. Rev. Nucl. Part. Sci. 50, 481 (2000).

3. M. Alford, A. Kaputsin, F. Wilczek Phys. Rev. D 59, 502 (1999).

4. O. Kaczmarek, J. Engels, F. Karsch, E. Laermann Nucl. Phys. Proc. Suppl. 83, 369 (2000).

5. S. Hands, I. Montvay, M. Oevers, L. Scorzato, J. Skullerud Nucl. Phys. Proc. Suppl. 457, 385 (2000).

6. S. Hands, J.B. Kogut, M. P. Lombardo, S.E. Morrison Nucl. Phys. B 558, 327 (1999).

7. I.M. Barbour, A.J. Bell Nucl. Phys. B 372, 385 (1992).

8. J. Kogut et. al. Nucl. Phys. B 225, 93 (1983).

9. P.E. Gibbs Phys. Lett. B 172, 53 (1986). 


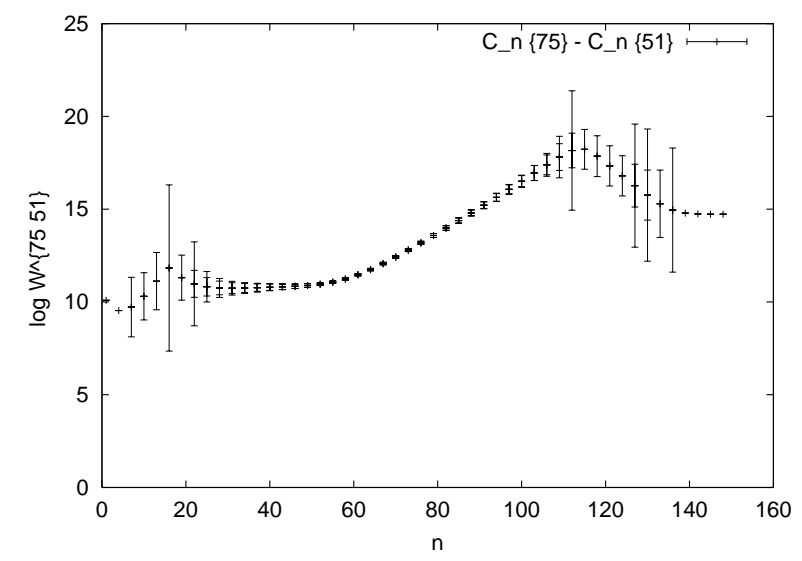

Figure 3. $\quad$ Log of the weighting factor ratio for static $\mathrm{SU}(3)$ ensembles generated using the Monte Carlo measures $c_{75}$ and $c_{51}$, defined through $W^{75}{ }^{51}(n)=\left\langle\frac{c_{n}}{c_{75}}\right\rangle_{c_{75}}\left(\left\langle\frac{c_{n}}{c_{51}}\right\rangle_{c_{51}}\right)^{-1}$.

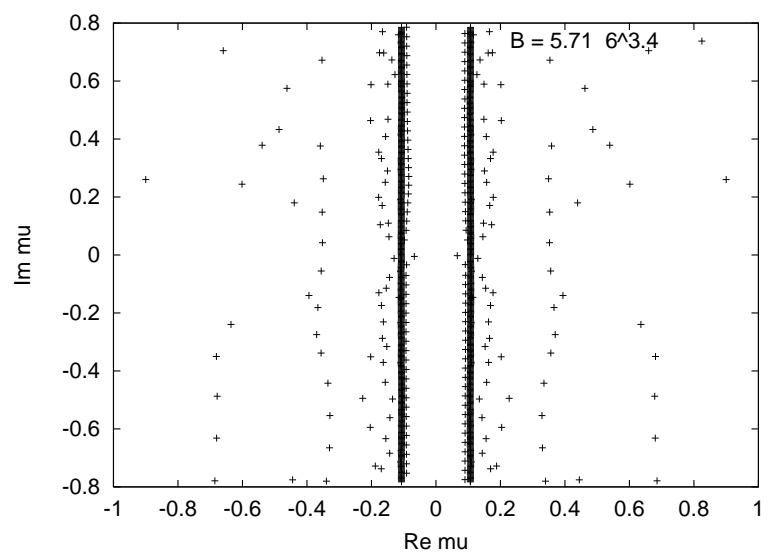

Figure 4. Lee Yang zeros evaluated in the complex $\mu$ plane from eleven composite weighted ensembles for static $\mathrm{SU}(3)$ at intermediate coupling.
10. C.N. Yang, T.D. Lee Phys. Rev. 87, 404:410 (1952).

11. C.T.H. Davies, E. Klepfish Phys. Lett. B 256, 68 (1991).

12. I.M. Barbour, J.B. Kogut, S.E. Morrison Nucl. Phys. B 53, 456 (1997).

13. M.A. Halasz, J.C. Osborn, M.A. Stephanov, J.J.M. Verbaarschot Phys. Rev. D 61, 76 (2000).

14. S. Chandrasekharan, U.J. Wiese Phys. Rev. Lett. 83, 3116 (1999).

15. S. Hands, J. Kogut, M.P. Lombardo, S.E. Morrison Nucl. Phys. B 558, 327 (1999).

16. E. Dagotto, F. Karsch, A. Moreo Phys. Lett. B 169, 421 (1986).

17. J.B. Kogut, D. Toublan, D.K. Sinclair heplat/0104010

18. B. Alles, M. D'Elia, M.P. Lombardo, M. Pepe Nucl. Phys. Proc. Suppl. 441, 395 (2001).

19. R. Aloisio, V. Azcoiti, G. Di Carlo, A. Galante, A. F. Grillo hep-lat/0011079

20. S.J. Hands, J.B. Kogut, S.E. Morrison, D.K. Sinclair Nucl. Phys. Proc. Suppl. 94457 (2001).

21. S. Hands, S. E. Morrison hep-lat/9905021

22. S. Morrison, S. Hands hep-lat/9902019

23. J.B. Kogut, D. Toublan, D. K. Sinclair heplat/0104010

24. M. P. Lombardo hep-lat/9907025

25. T. Blum and J. E. Terrick and D. Toussaint Phys. Rev. Lett. 76, 1019 (1996).

26. O. Kaczmarek, J. Engels, F. Karsch, E. Laermann Nucl. Phys. B 558, 307 (1999).

27. S. Chandrasekharan Nucl. Phys. Proc. Suppl. 9471 (2001).

28. I.M. Barbour, R. Buironi, G. Salina Phys. Lett. B 341, 355 (1995). 